\title{
High-Resolution MFM: Simulation of Tip Sharpening
}

\author{
Hitoshi Saito, Arnout van den Bos, Leon Abelmann, and J. Cock Lodder
}

\begin{abstract}
The transfer functions of tips with various sharpened tip ends were calculated and the resolution of these tips was estimated by considering the resolution limit due to thermal noise at room temperature. The tip having an ellipsoidal tip end (ellipsoidal tip) is found to be a suitable candidate for high-resolution magnetic force microscopy. Sharpening of the flat tip end makes zero signal frequencies disappear for tips with ellipticities larger than $\tan 45^{\circ}$. The sensitivity shows a maximum around an ellipticity of $\tan 80^{\circ}$. The ellipsoidal tip shows a much smaller tip thickness dependence compared to the tip having a flat tip end because only the tip end mainly contributes to signals in case of the ellipsoidal tip.
\end{abstract}

Index Terms-Magnetic force microscopy (MFM), magnetic tips, resolution, simulation.

\section{INTRODUCTION}

$\mathbf{M}$ AGNETIC FORCE microscopy (MFM) is widely used to study the microscopic magnetic domain structure of various magnetic thin films. The rapid decrease of the bit length in high-density magnetic recording requires a strong improvement of the spatial resolution of MFM to be able to observe the magnetic media and investigate nanoscale magnetism on magnetic films. There are many studies about high-resolution MFM tips, such as perforated tips [1], tips using electron beam-induced deposited carbon needles [2], focused ion beam modified tips [3], and CantiClever design [4] tips. The latter type features an elongated magnetic bar with flat tip end whose magnetization is parallel to the elongated bar direction. Among them, the CantiClever design [4] is expected to have the highest sensitivity and signal-to-noise ratio in the high-spatial frequency region, because the flat tip end ensures that the tip's magnetic charges are as close as possible to the sample, thereby maximizing the force exerted on the tip. A schematic picture of the CantiClever and a scanning electron microscope (SEM) image of the silicon nitride tip plane are depicted in Fig. 1.

The desired bar shape of the magnetic needle is obtained by deposition of a Co layer on the side of the freestanding SiN plane. To fabricate this type of cantilevers and use them for MFM observation, it is important to understand the influence

Manuscript received January 3, 2003. This work was supported in part by the Japanese Ministry of Education, Science, Sports and Culture under a Grant-in-Aid for Science Research and Overseas Research Scholarship.

H. Saito is with Akita University, Akita, Japan (e-mail: hsaito@ipc.akitau.ac.jp).

A. van den Bos, L. Abelmann, and J. C. Lodder are with the Systems and Materials for Information Storage Group, MESA ${ }^{+}$Research Institute, University of Twente, 7500 AE Enschede, The Netherlands (e-mail: a.g.vandenbos@el.utwente.nl; L.Abelmann@el.utwente.nl; J.C.Lodder@el. utwente.nl).

Digital Object Identifier 10.1109/TMAG.2003.816178
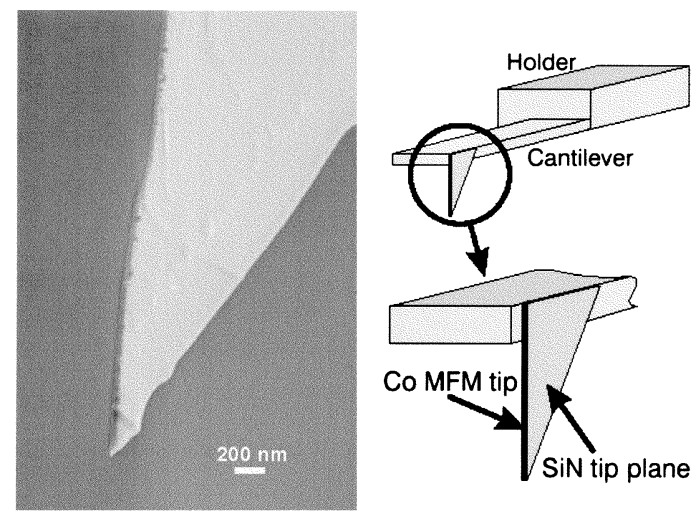

Fig. 1. SEM image and schematic picture of the CantiClever.

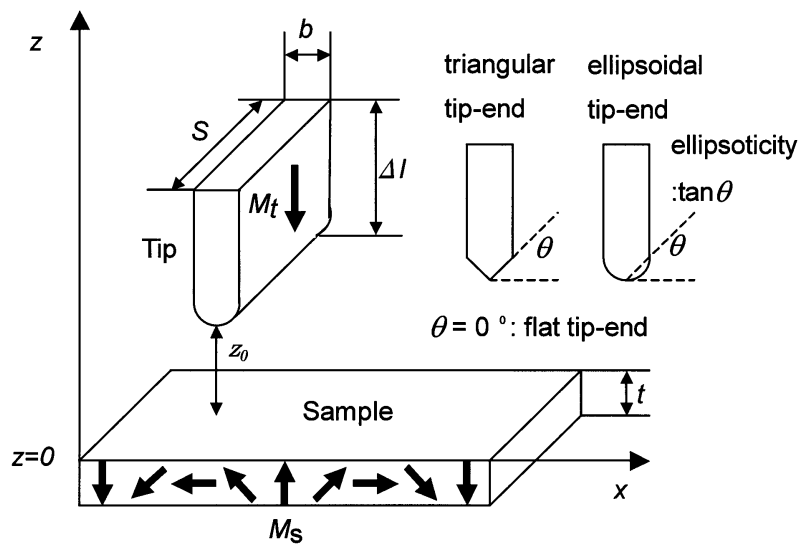

Fig. 2. Calculation modal of MFM signal for the CantiClever design.

of deviation on the ideal design, a flat tip end, such as the sharpening of the end of the tip. In this study, we calculate MFM signals for tips with various tip end shapes and discuss the effect of the tip sharpening on the sensitivity in MFM measurements and the maximum resolution that can be obtained.

\section{METHODS}

Fig. 2 shows the calculation model of MFM signals for the CantiClever design. The $z$ direction is defined perpendicular to the sample surface. A tip with its magnetization perpendicular to the sample surface $\left(M_{z}=M_{t}\right)$, a bar shape with thickness (magnetic film thickness) $b$, width (tip plane thickness) $S$, length (tip plane length) $\Delta l$, and a sample with perpendicular magnetization $\left.\left(M_{z}=M_{s} \cos \left(k_{x} x\right)\right)\right)$ and thickness $t$ is used to calculate the effect of changing the shape of the tip end. Here, no fluctuation of the tip and sample magnetization is considered. The MFM signal for a sample with a periodic 
magnetic surface charge density is calculated by using the following [5], [6]:

$$
\begin{aligned}
F_{z}^{\prime}= & -\frac{\partial^{2}}{\partial z^{2}} U=-\frac{\partial^{2}}{\partial z^{2}} \mu_{0} \int_{\text {tip }} M_{\text {tip }} H_{\text {sample }} d V_{\text {tip }} \\
= & -\frac{\partial^{2}}{\partial z^{2}} \mu_{0} \\
& \cdot \int_{\text {tip }} M_{t} \frac{M_{s}}{2} \cos \left(k_{x} x\right)\left(1-\mathrm{e}^{-k x t}\right) \mathrm{e}^{-k x z} d V_{\text {tip }} .
\end{aligned}
$$

The calculations were carried out for tips with three different tip-end shapes: flat, triangular, and ellipsoidal. From calculations with changing $k_{x}$, the MFM transfer function is then obtained using the following values (identical to those in [7]): $M_{t}=1422 \mathrm{kA} / \mathrm{m}(\mathrm{Co}), b=20 \mathrm{~nm}, S=100 \mathrm{~nm}, \Delta l=1 \mu \mathrm{m}$, $M_{s}=295 \mathrm{kA} / \mathrm{m}, t=70 \mathrm{~nm}$, and tip-sample distance $z_{0}$ is $10 \mathrm{~nm}$.

\section{RESULTS AND DISCUSSION}

From the present calculated results, the tip having an ellipsoidal shape is found to be the most suitable candidate for highresolution MFM among the three different shapes of tip ends. The ellipsoidal tip has higher sensitivity than the triangular tip. Fig. 3(a) shows the dependence of spatial frequency and tip angle on $\left|F_{z}^{\prime}\right|$ (absolute value of $F_{z}^{\prime}$ ) for ellipsoidal tips. Fig. 3(b) shows the spatial frequency dependence of $\left|F_{z}^{\prime}\right|$ (MFM transfer function) for ellipsoidal tips with several ellipticities. The MFM tip having a flat tip end $\left(\theta=0^{\circ}\right)$ corresponding to the CantiClever design has the highest sensitivity of all three types apart from zeros at certain spatial frequencies. The zero signal character arises from the same origin as those of gap zeros in magnetic recording heads. For the flat tip, zero signals occur when an integer multiple of a wavelength of the periodic magnetic stray field is within the tip thickness $b$. Moreover, the sign of $F_{z}^{\prime}$ changes alternately when spatial frequency crosses a certain zero frequency. The sign of $F_{z}^{\prime}$ is negative in the frequency region between the first and second zero. In this region, the total MFM signal is negative even when the sign of magnetic stray field is positive just under the tip center. Therefore, it is difficult to interpret the MFM signals containing wide spatial frequency components in case of the flat tip. When this tip is used in the frequency region below the first zero frequency, the resolution can be improved by reducing the thickness of the tip. As seen in Fig. 3(a), sharpening of the tip end makes the zero signal spatial frequencies disappear for tips with ellipticities larger than $\tan 45^{\circ}$. In the region with no zero frequencies, the sign of $F_{z}^{\prime}$ is always positive. The sensitivity shows a maximum around an ellipticity of $\sim \tan 80^{\circ}$.

Using the transfer function of the MFM tips, the resolution of the various tips is estimated by considering the resolution limit due to the thermal noise at room temperature [8]. The resolution can be estimated by the intersecting point between a certain transfer function and the noise level. This point represents the critical wavelength $\lambda_{c}$ [5]. As seen in Fig. 3(b), at the thermal noise level, the $\lambda_{c}$ of the ellipsoidal tip having an ellipticity of

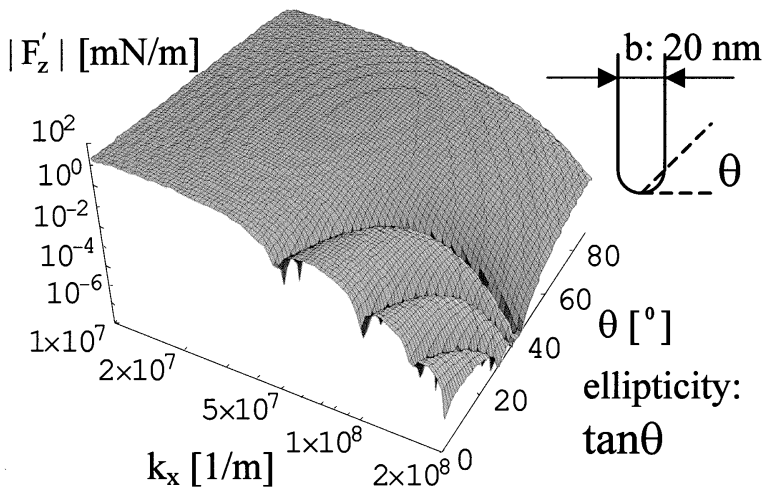

(a)

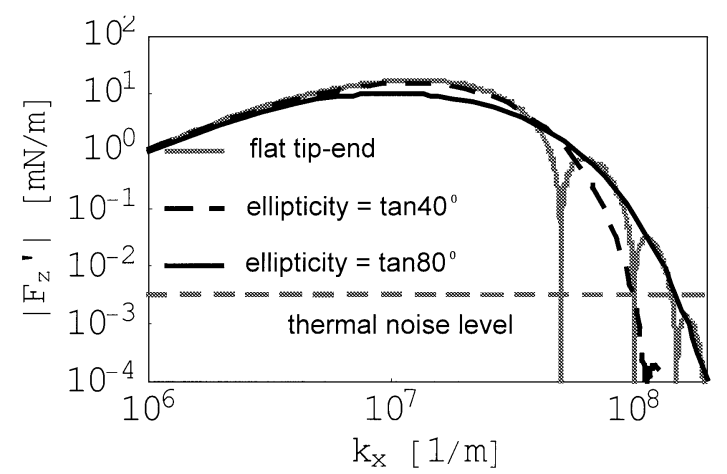

(b)

Fig. 3. (a) Dependence of spatial frequency and tip angle on $\left|F_{z}^{\prime}\right|$ for ellipsoidal tips ( $b=20 \mathrm{~nm}, z_{0}=10 \mathrm{~nm}$ ). (b) Spatial frequency dependence of $\left|F_{z}^{\prime}\right|$ for ellipsoidal tips with several ellipticities $\left(b=20 \mathrm{~nm}, z_{0}=10 \mathrm{~nm}\right)$.

$\sim \tan 80^{\circ}$ is the highest for the tips having tip thickness of $20 \mathrm{~nm}$.

These spatial frequency dependences of MFM signal for different types of tips is still the same with changing the tip-sample distance $z_{0}$ because the intersecting points among these curves in Fig. 3(b) do not change due to the character of MFM tip's transfer function [9], [10]. In the spatial frequency domain, the change of $z_{0}$ is equivalent to multiplying the term of $\exp \left(k_{x} \Delta z\right)$, where $\Delta z$ is the changed value of $z_{0}$ [9], [11]. Here, the positive and negative value of $\Delta z$ corresponds to the increase and decrease of $z_{0}$, respectively.

The dependence of $\lambda_{c}$ on the thickness of the tip is depicted in Fig. 4(a). Fig. 4(b) shows the signal density dependence on the position within the tip that has a thickness of $5 \mathrm{~nm}$ for a periodic magnetic field with a wavelength $=7 \mathrm{~nm}$. Here, $x=0$ corresponds to the center position of the tip. The MFM signal was obtained by integrating the signal density compared to the thickness of the tip.

As seen in Fig. 4(a), the ellipsoidal tip shows a much smaller dependence of $\lambda_{c}$ on the thickness of the tip than the flat tip and it also has a higher resolution than the flat tips for a tip thickness above $5 \mathrm{~nm}$. The reason for this is that only the center part of the tip end mainly contributes to the signal in case of the ellipsoidal tip as shown in Fig. 4(b). The general trend that the $\lambda_{c}$ of the ellipsoidal tip is smaller than that of the flat tip above the critical value of tip thickness is still the same with changing the tipsample distance $z_{0}$. This critical value increases with increasing $z_{0}$ and decreases with decreasing $z_{0}$. 


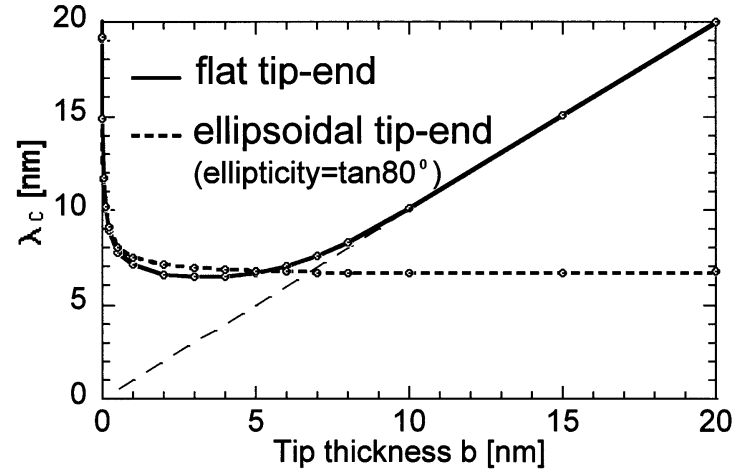

(a)

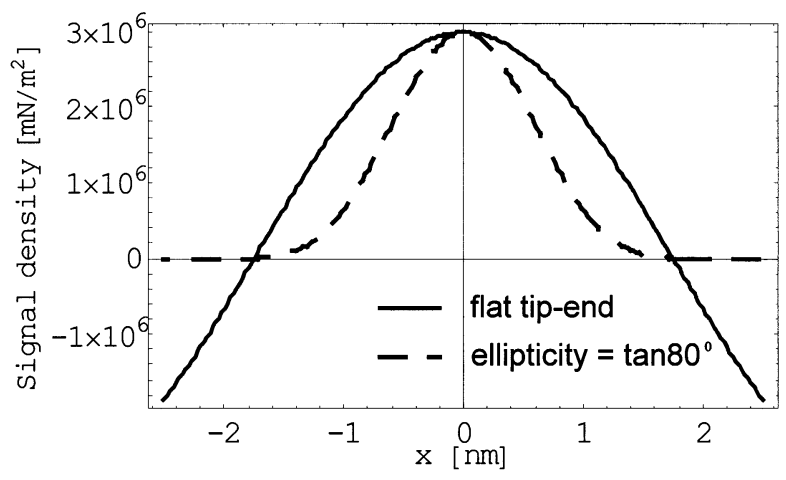

(b)

Fig. 4. (a) Tip thickness dependence of $\lambda_{c}$ for the tips with flat tip end and ellipsoidal tip end $\left(z_{0}=10 \mathrm{~nm}\right)$. (b) Signal density dependence on the position within the tip that has a thickness of $5 \mathrm{~nm}$ for a periodic magnetic field with a wavelength $=7 \mathrm{~nm}$.

From these results, it can be concluded that the degradation of the resolution due to the sharpening of the flat end of the tip is quite small in the high-resolution region. Therefore, in the limit where noise is only determined by cantilever thermal vibration, the ellipsoidal tip has a better resolution than the flat tip.

\section{CONCLUSION}

The transfer functions of tips with various sharpened tip ends were calculated and the resolution of these tips was estimated by considering the resolution limit due to thermal noise at room temperature. The tip having an ellipsoidal tip end is found to be a better candidate for high-resolution MFM, than the flat tip end suggested in [5]. The sharpening of the flat tip end makes zero signal frequencies disappear for tips with ellipticities larger than $\tan 45^{\circ}$ and the sensitivity shows a maximum around an ellipticity of $\tan 80^{\circ}$. The ellipsoidal tip shows a much smaller dependence of $\lambda_{c}$ on the thickness of the tip compared to the tip having a flat tip end because only the tip end mainly contributes to the signal for the ellipsoidal tip. Only for very high resolution, at a critical wavelength of about $7 \mathrm{~nm}$, does the flat tip end have some advantages. It should be noted, however, that the above results are valid at the thermal noise limit. In reality, additional noise sources (electronic noise, thermal drift, etc.) will increase the noise level and the crossover point in Fig. 4(a) will shift to thicker tips and higher critical wavelengths. Finally, in the CantiClever production process the tip will certainly become rounded off and resemble the ellipsoidal shape discussed in this paper. We now understand that this is a desirable effect and could explain why we observe resolutions better than $30 \mathrm{~nm}$ with 50-nm CantiClever tips [4].

\section{REFERENCES}

[1] L. Folks, M. E. Best, P. M. Rice, B. D. Terris, and D. Chapman, "Perforated tips for high-resolution in-plane microscopy," Appl. Phys. Lett., vol. 76, pp. 909-911, 2000.

[2] P. B. Fischer, M. S. Wei, and S. Y. Chou, "Ultrahigh resolution magnetic force microscope tip fabricated using electron beam lithography," J. Vac. Sci. Technol. B, vol. 11, pp. 2570-2573, 1993.

[3] G. N. Phillips, L. Abelmann, M. Siekman, and J. C. Lodder, "High resolution magnetic force microscopy using focused ion beam modified tips," Appl. Phys. Lett., vol. 81, pp. 865-867, 2002.

[4] A. van den Bos, I. Heskamp, M. Siekmann, L. Abelmann, and C. Lodder, "The CantiClever: a dedicated probe for magnetic force microscopy," IEEE Trans. Magn., vol. 38, pp. 2441-2443, Sept. 2002.

[5] S. Porthun, L. Abelmann, S. J. L. Vellekoop, C. Lodder, and H. J. Hug, "Optimization of lateral resolution in magnetic force microscopy," Appl. Phys. A, vol. 66, pp. S1185-S1189, 1998.

[6] S. Porthun, L. Abelmann, and C. Lodder, "Magnetic force microscopy of thin film media for high density magnetic recording," J. Magn. Magn. Mater, vol. 182, pp. 238-273, 1998.

[7] L. Abelmann, S. Porthun, M. A. M. Haast, J. C. Lodder, A. Moser, M. E. Best, P. J. A. Schendel, B. Stiefel, H. J. Hug, G. P. Heydon, A. Farley, S. R. Hoon, T. Pfaffelhuber, R. Proksch, and K. Babcock, "Comparing the resolution of magnetic force microscopes using the CAMST reference samples," J. Magn. Magn. Mater., vol. 190, pp. 135-147, 1998.

[8] T. R. Albrecht, P. Grutter, D. Horne, and D. Rugar, "Frequency modulation detection using high-q cantilevers for enhanced force microscope sensitivity," J. Appl. Phys., vol. 69, pp. 668-673, 1991.

[9] H. J. Hug, B. Stiefel, P. J. A. Schendel, A. Moser, R. Hofer, S. Martin, H.-J. Guntherodt, S. Porthun, L. Abelmann, C. Lodder, G. Bochi, and R. C. O'Handley, "Quantitative magnetic force microscopy on perpendicular magnetic samples," J. Appl. Phys., vol. 83, pp. 5609-5620, 1998.

[10] H. Saito, J. Chen, and S. Ishio, "Description of magnetic force microscopy by three-dimensional tip Green's function for sample magnetic charges," J. Magn. Magn. Mater., vol. 191, pp. 153-161, 1999.

[11] - ,Principle of magnetic field analysis by MFM signal transformation and its application to magnetic recording media," IEEE Trans. Magn., vol. 35, pp. 3992-3994, Sept. 1999. 